\title{
Biomechanical Comparison of Krackow and Uchiyama Suture Techniques for Acute Tendon Repair in an Ex Vivo Porcine Model
}

\author{
Mary Rose C Gonzales ${ }^{1}$, Kristine R Italia ${ }^{2}$, Jose Raul C Canlas ${ }^{3}$, Richard S Rotor ${ }^{4}$, Maria Adelwisa G Belen ${ }^{5}$
}

\begin{abstract}
Aim and objective: This study compared the maximum load to failure of Krackow and Uchiyama suture techniques in an acute tendon repair in an ex vivo porcine model.

Background: Achilles tendon rupture is a common injury in athletes and active individuals. In 2007, Uchiyama et al. published a case series of a modified operation for Achilles tendon ruptures. This technique is reported to allow strong and stable repair with subsequent early weight bearing and range of motion exercises. However, no biomechanical studies have been performed to compare this novel suturing technique to a standard like Krackow.

Materials and methods: Twenty freshly harvested porcine digital flexor tendons were transected transversely at the mid-substance. Ten were repaired with double-strand Krackow suture technique using non-absorbable polyester braided suture Ethibond ${ }^{\oplus} 2$. The other 10 tendons were further divided into bundles approximately $5 \mathrm{~mm}$ wide -2 proximal and 2 distal. The Uchiyama repair was completed by gathering each bundle by Bunell-like sutures using Ethibond ${ }^{\oplus} 2-0$. Biomechanical testing was conducted on the samples using a mechanical universal testing machine (Shimadzu ${ }^{\oplus}$ AGS-X Series). Longitudinal traction at an advancement rate of $0.85 \mathrm{~mm} / \mathrm{second}$ was performed and maximum load to failure and mode of failure were statistically compared.
\end{abstract}

Results: The average maximum load to failure was not significantly different between the Krackow repair (119.18 N \pm 37.4$)$ and Uchiyama $(112.98 \pm 32.06)(p=0.34)$. All modes of failure were due to suture breakage.

Conclusion: There is no significant difference between the maximum load to failure of Uchiyama and Krackow suture technique.

Significance: Uchiyama suture technique may be an alternative to open repair of acute tendon of Achilles rupture. This warrants further studies to investigate other properties like failure to cyclic loading, tendon gapping, etc. In vivo studies may be geared toward effect of greater surface contact and smaller caliber sutures in tendon healing.

Keywords: Krackow technique, Laboratory research, Tendon repair, Tensile strength, Uchiyama technique.

Journal of Foot and Ankle Surgery (Asia Pacific) (2021): 10.5005/jp-journals-10040-1137

\section{INTRODUCTION}

Achilles tendon rupture is a common injury, which occurs not only to athletes but also in active individuals from 30 to 60 years of age. There are several studies performed evaluating which types of sutures are the strongest to be used for repair. As for the suture techniques, abundant researches are discussing Krackow, Bunnell, Kessler, triple bundle, and even the percutaneous Achillon (Integra) method device. In 2007, Uchiyama et al. published a case series of a modified repair for Achilles tendon ruptures. This new technique is reported to allow strong repair stability and subsequent early weight bearing and range of motion (ROM) exercises. ${ }^{1}$ However, to date, no biomechanical studies have been performed to compare this novel suturing technique to a standard like Krackow. This study compared the biomechanical property-maximum load to failure, of Krackow and Uchiyama tendon repair techniques and noted its mode of failure in an acute tendon rupture in an ex vivo porcine model.

\section{Materials and Methods}

Twenty freshly harvested porcine flexor digital tendons from the hind leg were used for this biomechanical experimental study. The semi-flat shape and bulk of the proximal end of porcine flexor digital tendons before branching out distally were comparable
${ }^{1-5}$ Department of Orthopedics, Institute of Orthopedics and Sports Medicine, St Luke's Medical Center, Quezon City, Philippines

Corresponding Author: Mary Rose C Gonzales, Department of Orthopedics, Institute of Orthopedics and Sports Medicine, St Luke's Medical Center, Quezon City, Philippines, Phone: +639171490921, e-mail:yosgonzales@gmail.com

How to cite this article: Gonzales MRC, Italia KR, Canlas JRC, et al. Biomechanical Comparison of Krackow and Uchiyama Suture Techniques for Acute Tendon Repair in an Ex Vivo Porcine Model. J Foot Ankle Surg (Asia Pacific) 2021;8(1):8-11.

Source of support: Nil

Conflict of interest: None

to the size of human adult Achilles tendons, which have a $5.1 \mathrm{~mm}$ mean thickness and $117.7 \mathrm{~mm}$ length determined sonographically. ${ }^{2}$ This dimension allowed accommodating the suture size and configuration used commonly for repair of larger tendons. Tendons were harvested within 6 hours after death of the animal. Harvested tendons were wrapped in saline-soaked gauze then refrigerated at $4^{\circ} \mathrm{C}$ after removal of extraneous muscle tissues. Maximum storage time was limited to 12 hours in the portable mini-refrigerator. Harvested tendons were randomly assigned to each of the 2 groups, with 10 tendons per group. ${ }^{3,4} \mathrm{~A}$ scalpel was used to transect each 


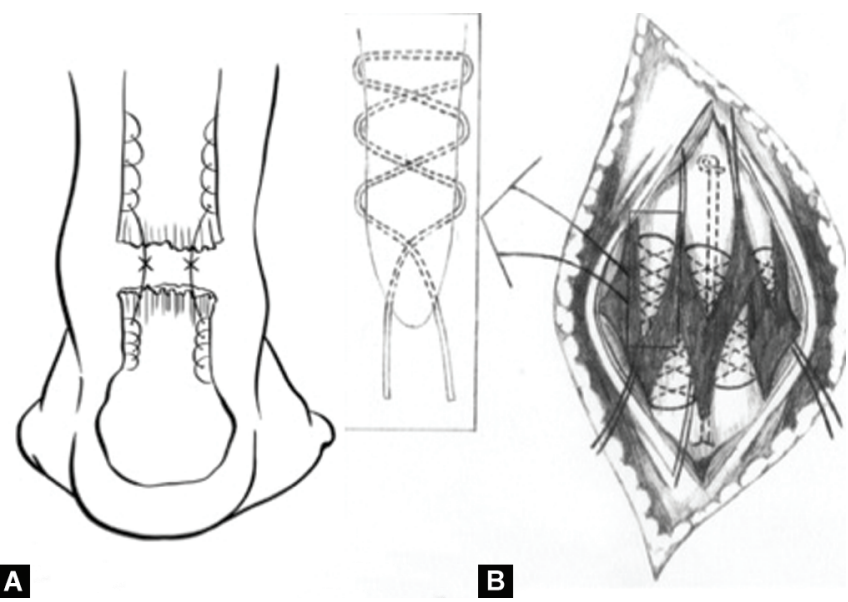

Figs $1 \mathrm{~A}$ and $\mathrm{B}$ : (A) Krackow technique; (B) Uchiyama technique ${ }^{1,5}$

of the tendons transversely at the mid-substance, immediately preceding the repair. Individual samples were then shortly returned to storage after preparation to prevent desiccation. A single investigator performed the harvest, preparation, and repair of all tendons.

Repair was performed using Krackow technique with a nonabsorbable braided suture Ethibond ${ }^{\otimes} 2$ on the first group, and Uchiyama technique with Ethibond ${ }^{\oplus} 2-0$ on the second group. Using a double-strand Krackow technique, four locking loops were spaced $5 \mathrm{~mm}$ from each other, $1 \mathrm{~cm}$ from the proximal end of the tendon. Each locking stitch was tensioned separately to avoid excess suture material within the loops. The sutures were tied with one surgeon's knot and four simple half-hitches (Fig. 1A). ${ }^{4,5}$ For the Uchiyama technique, each tendon end was divided into bundles approximately $5 \mathrm{~mm}$ wide -2 proximal and 2 distal. The reconstruction was completed by gathering each bundle by Bunnell-like sutures. Each bundle was pulled lightly and fixed to the other end of the tendon in a linear fashion-proximal bundles to the distal end, and vice versa (Fig. 1B). ${ }^{1}$

Biomechanical testing was conducted by a single civil engineer using a mechanical universal testing machine (Shimadzu ${ }^{\circledast}$ AGS-X Series Dual Column Electromechanical Test Frame, North America), with each end of a sample tendon secured using metal clamps. Load to failure test was performed with longitudinal traction at an advancement rate of $0.85 \mathrm{~mm} / \mathrm{second} .{ }^{4}$ Failure is defined as suture breakage and pull-through or pull-out, until no further increase in the load registered on the machine, indicating that the ultimate load has been reached. Ultimate load to failure (in Newton) was measured. Mode of failure was recorded, whether at the suture or tendon substance ${ }^{3,4}$ (Fig. 2 and Table 1).

\section{Results}

Both suture techniques had similar maximum load to failure [119.18 $\mathrm{N} \pm 37.4$ (Krackow) vs $112.98 \pm 32.06$ (Uchiyama), $p=0.34$ ] ( $t$-test, SPSS $2017^{\oplus}$ ). All modes of failure were due to suture breakage.

\section{Discussion}

Six out of 100,000 people, with middle-aged athletes as the most commonly affected, suffer from a rupture of their Achilles tendon per year. This concern is not only limited to athletes but includes the active elderly. Currently, Achilles tendon ruptures are dealt with surgical intervention in the active population. The primary goal

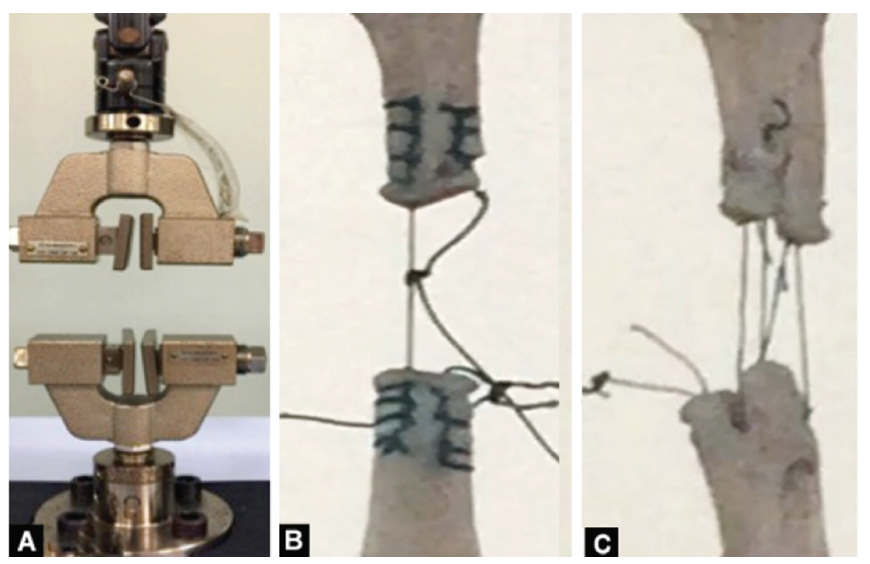

Figs 2A to C: (A) Biomechanical testing conducted on the samples using a mechanical universal testing machine (Shimadzu ${ }^{\circledR}$ AGS-X Series); Longitudinal traction at an advancement rate of $0.85 \mathrm{~mm} / \mathrm{second}$ was done and maximum load to failure was noted on both (B) Krackow and (C) Uchiyama repair ${ }^{1,3,4}$

Table 1: Load to failure (Newton) of porcine tendons repaired by Krackow and Uchiyama technique

\begin{tabular}{clcll}
\hline $\begin{array}{l}\text { Maximum load } \\
\text { to failure }(\mathrm{N})\end{array}$ & $\begin{array}{l}\text { Tendon } \\
\text { sample }\end{array}$ & $\begin{array}{l}\text { Maximum load } \\
\text { to failure }(\mathrm{N})\end{array}$ & \\
\hline Krackow & 135.75 & 1 & 108.13 & Uchiyama \\
137.50 & 2 & 75.62 & \\
126.88 & 3 & 75.00 & \\
137.50 & 4 & 101.87 & \\
135.62 & 5 & 139.375 & \\
128.30 & 6 & 156.87 & \\
98.00 & 7 & 140.01 & \\
176.00 & 8 & 133.09 & \\
63.60 & 9 & 66.99 & \\
52.65 & 10 & 132.86 & \\
$119.18 \mathrm{~N} \pm 37.4$ & $p=0.34$ & $112.98 \pm 32.06$ & \\
& $(t$-test $)$ & &
\end{tabular}

of surgical reconstruction and the crucial determinant of surgical success remain the initial strength and optimum length of the Achilles tendon repair. ${ }^{6}$

No single technique has been absolutely shown to be superior to others. A biomechanical systematic study of repair in cadaveric human Achilles tendons concluded that a definitive recommendation on the most optimal repair technique still cannot be made due to subject heterogeneity and varied methodology. ${ }^{6}$ Having a relatively new tendon repair technique, it is vital to know its biomechanical properties and compare it to the standard to allow optimal use in the clinical setting.

Different suture techniques ranging from Krackow, bunnell, kessler, triple bundle, and even the percutaneous Achillon (Integra) method device have been repeatedly studied with no definitive recommendation on the most optimal repair technique. Two studies with a total of 27 ankles reported that Krackow sutures had a significantly better outcome $(1.37,95 \% \mathrm{Cl}=2.286$ to 0.468 , $l^{2}=83.4 \%, p=0.003$ ) compared to kessler. ${ }^{7}$ The number of locking loops used might have had an influence on the strength of the Krackow suture configuration using the larger diameter, 
high-strength sutures. ${ }^{8}$ It has been shown in a load to failure test that the Krackow locking-loop suture had a pull-out strength of 392 $\mathrm{N}$ and greater axial resistance than open repairs, such as the Bunnell and Kessler repair. ${ }^{3}$ Lastly, in a systematic review of 11 studies, triple bundle technique in combination with Ethibond ${ }^{\oplus} 2$ performing best with a mean of $453 \mathrm{~N}$. Recommendations were not made due to small sample size, different study designs, and heterogeneity of measurement techniques. ${ }^{6}$ However, they agree to support the wide use of a double-bundle Krackow suture technique utilizing Ethibond ${ }^{\oplus}$ sutures as repair option for acute Achilles tendon rupture.

In 2007, Uchiyama et al. published a case series of a modified operation for Achilles tendon ruptures. This new technique is reported to allow strong repair stability and subsequent early weight bearing and ROM exercises. Together with a unified rehabilitation protocol, this allowed early weight bearing and return to daily activities. Tendon repair was performed as follows: Each tendon end was divided into bundles approximately $5 \mathrm{~mm}$ wide; although often 3 proximal bundles and 2 distal bundles, these could be 2 proximal and 3 distal, or 2 proximal and 2 distal, according to the original condition of the ruptured tendon. The reconstruction was completed by gathering each bundle by Bunnell-like sutures, using a non-absorbable polyester braided suture (Tevdek ${ }^{\circledR} 2-0$, Teleflex Medical). Each bundle was pulled lightly and fixed to the other end of the tendon in a linear fashion-proximal bundles to the distal end, and vice versa. ${ }^{1}$

With standard methods of surgical treatment of acute Achilles tendon rupture, patients are frequently placed in a cast for 6 weeks and not allowed full weight bearing up to 2 weeks. However, longer immobilization is thought to have detrimental postoperational effects while early full weight bearing is believed to increase the risk of rerupture. ${ }^{9}$

In Uchiyama's method, they adapted shorter duration of immobilization ${ }^{9}$ and limited weight bearing. Patients wore a cast for 2 weeks, but were allowed full weight bearing gait at 1 week postoperatively. Although average ROM recovery was at 10 weeks, $92 \%$ recovered their original ROM in 17 weeks. They were able to do double-legged heel raises in an average of 7.6 weeks and singlelegged heel raises in 12 weeks. Twenty-one high-level athletes returned to their original game level at an average of 5 months. These results are comparable with those mentioned in past reports about early postoperative rehabilitation. Bearing weight on the operated leg not only leads to quicker return to daily activities but also trains the muscles on the operated side and prevents articular cartilage deterioration. Since full weight bearing is allowed from day 7 , this will allow earlier functional recovery and a quicker return to sports. ${ }^{1}$

The repair strength must (1) withstand the high forces applied by early active mobilization that is important to obtain good functional tendon glide and (2) avoid early rupture and adhesion formation. Both are vital toward optimal tendon healing and early return to function. ${ }^{10}$

In this study, Uchiyama technique had a similar repair strength based on maximum load to failure despite the less tensile strength of Ethibond 2-0 (0.3 mm suture diameter) compared to Ethibond 2 ( $0.5 \mathrm{~mm}$ suture diameter). This may be attributed to Uchiyama having 8 core sutures vs the 2 core sutures of Krackow repair. A $50 \%$ increase in strength to failure would be expected by increasing the number of crossing strands by 50\%. ${ }^{11}$ Specifically, a 12 core repair using a 2-0 non-absorbable suture was stronger than a 6 core repair using a 2 non-absorbable suture with mean ultimate tensile strength of $828 \mathrm{~N}$ and $438 \mathrm{~N}$, respectively. ${ }^{12}$ However, multistrand core repair techniques are stronger and gap resistant but may be detrimental to glide and cause increased tissue trauma. ${ }^{10}$

With similar maximum load to failure, this may facilitate earlier ROM for both Uchiyama and Krackow techniques of $<2$ weeks. ${ }^{10}$ Immobilized tendon sutures lose $50 \%$ of their initial strength within the first week due to tenomalacia at the suture-tendon junction. Early passive and active rehabilitation programs have been shown to prevent initial weakening at the repair site by improving tendon nutrition, healing, and remodeling. ${ }^{13}$ Controlled mobilization after the 1-week inflammatory phase postinjury enhances the quality of healing tendons by stimulating fibroblast proliferation and collagen realignment. $^{14}$

\section{Limitations}

This study reports the maximum load to failure of the Uchiyama and aims to support its assertion of strong repair stability and subsequent early weight bearing and ROM exercises with no rerupture. However, being an ex vivo biomechanical study, the injury was created using a sharp scalpel rather than the degenerative tendon tissue associated with clinical rupture. The tendon repair was also performed at time zero from injury. ${ }^{15}$ This may oversimplify Achilles tendon repair postoperative rehabilitation. Data gathered are limited to ultimate load to failure and may not be comparable to hypothesized earlier ROM and weight bearing after surgery. ${ }^{12}$

\section{Conclusion and Clinical Significance}

There is no significant difference between the load to failure of Uchiyama and Krackow suture technique. Uchiyama suture technique may be an alternative to open repair of acute tendon of Achilles rupture. However, further studies are recommended to investigate other biomechanical properties, such as failure to cyclic loading and tendon gapping. In vivo studies may also be performed to investigate other properties of the Uchiyama technique like effect of greater surface contact and smaller caliber sutures in tendon healing.

\section{References}

1. Uchiyama E, Nomura A, Takeda Y, et al. A modified operation for Achilles tendon ruptures. Am J Sports Med 2007;35(10):1739-1743. DOI: 10.1177/0363546507304492.

2. Pang B, Ying M. Sonographic measurement of Achilles tendon in asymptomatic subjects variations with age body height and dominance of ankle. J Ultras Med 2006;25(10):1291-1296. DOI: 10.7863/jum.2006.25.10.1291.

3. Ortiz C, Wagner E, Morocain P, et al. Biomechanical comparison of four methods of repair of the Achilles tendon a laboratory study with bovine tendons. J Bone Joint Surg 2012;94(5):663-667. DOI: 10.1302/0301-620X.94B5.27642.

4. Tio Cuison J, Tablante E. Ex vivo biomechanical analysis of barbed suture tenorraphy in a porcine model. Presented in Asia-pacific knee arthroscopy and sports medicine society summit Seoul, Korea. April 28, 2017.

5. Watson T, Jurist K, Yang K, et al. The strength of Achilles tendon repair: an in vitro study of the biomechanical behavior in human cadaver tendons. Foot Ankle Int 1995;16(4):191-195. DOI: 10.1177/107110079501600404.

6. Sadoghi P, Rosso C, Valderrabano V, et al. Initial Achilles tendon repair strength - synthesized biomechanical data from 196 cadaver repairs. Int Orthoped 2012;12(9):1533-1536. DOI: 10.1007/s00264-0121533-6. 
7. Yammine K, Assi C. The efficacy of repair techniques of the Achilles tendon. A meta-analysis of human cadaveric biomechanical studies. The Foot 2017;30:13-20. DOI: 10.1016/j.foot.2016.09.006.

8. Hapa O, Erduran M, Havitcioglu H. Strength of different Krackow stitch configurations using high-strength suture. J Foot Ankle Surg 2013;52(4):448-450. DOI: 10.1053/j.jfas.2013.03.009.

9. American Association of Orthopedic Surgeons. The diagnosis and treatment of acute Achilles tendon rupture: guideline and evidence report. 2009 [cited 2018] Available from: http://www.aaos.org/ research/guidelines/guide.asp.

10. Rawson S, Cartmell S, Wong J. Suture techniques for tendon repair; a comparative review. Muscle, Ligam Tendons J 2013;3(03):220-228. DOI: 10.32098/mltj.03.2013.16.

11. Hu'ffard B, O'Loughlin P, Wright $\mathrm{T}$, et al. Achilles tendon repair: Achillon system vs. Krackow suture: an anatomic in vitro biomechanical study. Clin Biomechan 2008;23(9):1158-1164. DOI: 10.1016/ j.clinbiomech.2008.05.007.

12. Backus J, Marchetti D, Slette $E$, et al. The effect of suture caliber and number of core strands on repair of acute Achilles ruptures using a mini-open repair: a biomechanics study. Am Orthoped Foot Ankle Soc 2017;38(5):564-570. DOI: 10.1177/1071100716687368.

13. Clemente A, Bergamin F, Surace C, et al. Barbed suture vs conventional tenorrhaphy: biomechanical analysis in an animal model. J Orthopae Traumatol 2015;16(3):251-257. DOI: 10.1007/s10195-0140333-8.

14. Wang J. Mechanobiology of tendon. J Biomechanics 2006;39(9): 1563-1582. DOI: 10.1016/j.jbiomech.2005.05.011.

15. Wu Z, Hua Y, Li H, et al. Biomechanical comparison of three methods for distal Achilles tendon reconstruction. Knee Surg Sports Traumato Arthrosc 2015;23(12):3756-3760. DOI: 10.1007/s00167-014-3176-1. 